\title{
Overcoming cellular senescence in human cancer pathogenesis
}

Thomas R. Yeager, ${ }^{1,6}$ Sandy DeVries, ${ }^{10}$ David F. Jarrard, ${ }^{1,4,5}$ Chinghai Kao, ${ }^{9}$ Stephen Y. Nakada, ${ }^{4,5}$ Timothy D. Moon, ${ }^{4,5}$ Reginald Bruskewitz, ${ }^{4,5}$ Walter M. Stadler, ${ }^{8}$ Lorraine F. Meisner, $^{7}$ Kennedy W. Gilchrist, ${ }^{3,5}$ Michael A. Newton, ${ }^{2}$ Frederic M. Waldman, ${ }^{10}$ and Catherine A. Reznikoff ${ }^{1,5,6,11}$

\begin{abstract}
Departments of ${ }^{1} \mathrm{H}$ uman Oncology, ${ }^{2}$ Biostatistics, ${ }^{3}$ Pathology, and ${ }^{4}$ Surgery, ${ }^{5}$ University of Wisconsin Comprehensive Cancer Center and University of Wisconsin Medical School, ${ }^{6}$ Program in Cellular and Molecular Biology, and ${ }^{7}$ State Laboratory of Hygiene, Madison, Wisconsin 53792 USA; ${ }^{8}$ Department of Hematology/Oncology, University of Chicago, Chicago, Illinois 60637 USA; ${ }^{9}$ Department of U rology, University of Virginia, Charlottesville, Virginia 22908 USA;

${ }^{10}$ Department of Laboratory Medicine and Division of M olecular Cytometry, University of California at San Francisco, San Francisco, California 94143 USA
\end{abstract}

Elevation of p16, the CD KN 2/p16 tumor suppressor gene (TSG) product, occurs at senescence in normal human uroepithelial cells (HUC). Immortal HUCs and bladder cancer cell lines show either alteration of p16 or pRb, the product of the retinoblastoma (RB) TSG. In addition, many human cancers show p16 or pRb alteration along with other genetic alterations that we associated with immortalization, including $+20 \mathrm{q}$ and $-3 p$. These observations led us to hypothesize that p16 elevation plays a critical role in senescence cell cycle arrest and that overcoming this block is an important step in tumorigenesis in vivo, as well as immortalization in vitro. Using a novel approach, we tested these hypotheses in the present study by examining p16 and pRb status in primary culture (P0) and after passage in vitro of transitional cell carcinoma (TCC) biopsies that represented both superficial bladder tumors and invasive bladder cancers. We demonstrated that all superficial TCCs showed elevated p16 after limited passage in vitro and then senesced, like normal HUCs. In contrast, all muscle invasive TCCs contained either a p16 or a pRb alteration at P0 and all spontaneously bypassed senescence $(P=0.001)$. Comparative genomic hybridization $(C G H)$ was used to identify regions of chromosome loss or gain in all TCC samples. The application of a statistical model to the CGH data showed a high probability of elevated alteration rates of +20q11-q12 (0.99) and +8p22-pter (0.94) in the immortal muscle invasive TCCs, and of $-9 q(0.99)$ in the superficial TCCs. Three myoinvasive TCCs lost 3p13-p14. In this study, four of six myoinvasive TCCs also showed TP53 mutation that associated well with genome instability $(P=0.001)$, as previously hypothesized. Notably, T P53 mutation, which has been used as a marker of tumor progression in many human cancers, was less significant in associating with progression in this study $(P=0.04)$ than was p16 or pRb alteration $(P=0.001)$. Thus, these data support a new model in which overcoming senescence plays a critical role in human cancer pathogenesis and requires at least two genetic changes that occur in several combinations that can include either p16 or pRb loss and at least one additional alteration, such as $+20 q 11-q 12,-3 p 13-p 14$, or -8p21-pter.

[Key Words: Senescence; cancer progression; p16; pRb; p53; +20; -3p; -8p]

Received September 10, 1997; revised version accepted N ovember 11, 1997.

Our laboratory has a longstanding interest in studying the biological significance of genetic alterations in the pathogenesis of human cancer. The identification of alterations that are associated specifically with progression to muscle-invading (myoinvasive) cancer will be particularly useful for diagnosis, prognosis, and treatment. Many studies suggest that p53 mutation might represent such an alteration because of its prevalence across many human cancer types and its putative role in

${ }^{11}$ Corresponding author.

E-MAIL CRezniko@FACSTAFF.wisc.edu; FAX (608) 262-8430. maintenance of genome stability (for review, see Reznikoff et al. 1996a). In the present study, using bladder cancer progression as a model, we test a new and possibly complementary model of human cancer pathogenesis. In this model, overcoming the $G_{1}$ growth arrest of senescence is a critical and essential step in the development of human carcinoma.

Bladder cancer is the sixth most common cancer in the United States and the third most preval ent cancer among men 60 years of age or older (American Cancer Society 1997). Causative factors known to significantly increase bladder cancer risk include smoking and exposure to ar- 
ylamine carcinogens (Kannio et al. 1996). A pproximately $90 \%$ of bladder cancers are transitional cell carcinomas (TCC), $80-90 \%$ of TCCs grow as superficial papillary stages Ta and T1 [T N M classification] tumors (Catal ona 1992; Bane et al. 1996). Approximately $60 \%$ of superficial TCCs recur, but most never progress to muscle invasive cancer and thus their associated mortality is low. However, $20-25 \%$ of superficial TCCs progress over time to myoinvasi ve TCCs that pose a significant risk of morbidity and mortal ity for patients ( $\mathrm{N}$ seyo and Lamm 1996). To illustrate the associated clinical dilemma, one study shows that 13 of 62 patients (21\%) diagnosed initially with superficial Ta or T1 well-differentiated grade I TCC eventually died of muscle invasive bladder cancer (Bane et al. 1996). Thus, there is a pressing need to understand the pathogenesis of progression of superficial TCC to myoinvasive TCC and to identify genetic biomarkers to stratify TCCs for appropriate clinical management.

$M$ any genetic al terations have been identified in bladder cancers, but the biological significance of most of these alterations has not been defined. For example, comparative genomic hybridization $(\mathrm{CGH})$ and molecuIar studies show that regions frequently $(>20 \%)$ lost include 3p14, 8p21, 9p21, 9q, 11p15, 13q14, and 17p13; frequent $(>14 \%)$ gains occur at $1 q 31,3 q 24-26$ and $8 q 21-$ qter (Hubuchi et al. 1993; Knowles et al . 1994; Kallioniemi et al. 1995). Amplifications have also been observed at 5p21, 11q13, 17q12, and 20q11-qter (Kallioniemi et al. 1995). Certain losses, including 3p14, 8p21, 11p15, 13q14, and 17p13, correlate with late stage disease (Presti et al. 1991; Hubuchi et al. 1993; Knowles et al. 1994). In contrast, loss of $9 q$ is grade and stage independent and, therefore, is thought to occur early (Ross et al. 1996). In general, losses are thought to indicate tumor suppressor gene (TSG) del etions, whereas gains indicate overrepresentation of oncogenes. Much evidence supports a role for inactivation of three TSG in TCC pathogenesis. These include the retinoblastoma gene (RB) at $13 q 14$, CDKN2/p16 at 9p21, and TP53 at 17p13 (Presti et al. 1991; Xu et al. 1993; Esrig et al. 1994; Spruck et al. 1994; Cairns et al. 1995). Some evidence also suggests that overexpression or amplification of the cyclin D1 gene CCND1 at 11q13 may also play a role in bladder cancer pathogenesis (Ohta et al. 1997; Shin et al. 1997).

Alteration of TP53, which occurs in $~ 50 \%$ of TCCS, has been associated with a genome instability phenotype in normal uroepithelium (Reznikoff et al. 1994) and is frequently present in muscle-invading TCC (Hollstein et al. 1991; Presti et al. 1991; Sarkis et al . 1993; Spruck et al. 1994a; Esrig et al. 1994). N otably, $60 \%$ of bladder carcinoma in situ (CIS), which have a high propensity for progression, show TP53 alterations, whereas only 3\% of superficial papillary TCCs show altered TP53 (Spruck et al. 1994a). Thus, TP53 alteration has been considered a useful genetic biomarker to predict TCC progression (Sarkis et al. 1993; Esrig et al. 1994). RB is inactivated in $30-40 \%$ of bladder cancers and this al so correlates with later stage, higher grade cancers (Presti et al 1991; Xu et al. 1993). RB plays a critical role in regulating cell cycle progression by its phosphorylation, which releases E2F transcription factors, a process that is tightly controlled by cyclin D1 and cdk4/6 (Xu et al. 1993), and which is inhibited by p16 (Serrano et al. 1993).

Defining the bi ol ogical significance of p16 loss in TCC pathogenesis has been problematic for several reasons. First, until recently (see bel ow) the role of p16 in normal human uroepithelial cell (HUC) growth has not been understood. In addition, although $\sim 50 \%$ TCC s show 9p21/ CDKN2/p16 hemizygous deletion, a much lower percent show inactivating mutations on the remaining allele (Cairns et al. 1994). Furthermore, a higher percentage of these mutations were observed in TCC cell lines than in uncultured tumors (Kamb et al. 1994). These results raised the question of the relevance of p16 loss to TCC tumorigenesis in vivo. An epigenetic mechanism for CDKN2/p16 inactivation, namely hypermethylation, has now been documented in many tumor types, including TCCs (Gonzalez-Zulueta et al. 1995, Merlo et al. 1995). In spite of these findings, the biological significance of p16 loss in the pathogenesis of TCC and other human cancers still remains unclear.

Recently, our laboratory reported that, whereas p16 levels are low to undetectable in precrisis proliferating HUCs, they are el evated at senescence (Reznikoff et al. 1996b). On the basis of this observation, we hypothesized that p16 may play a role in mediating the $\mathrm{G}_{1}$ cell cycle arrest that is characteristic of cellular senescence (Serrano et al. 1997). This model suggests that senescence is a tumor suppressor mechanism in vivo and that p16 is important in the onset and maintenance of senescence. Because the growth-suppressive function of p16 requires a functional $\mathrm{pRb}$ (Lukas et al. 1995; M edema et al. 1995), this model al so suggests that overcoming the p16-imposed component of cellular senescence could be accomplished by either p16 or pRb al teration. This hypothesis is supported by our finding that all immortal HUC and TCC cell lines tested to date that do not contain a p16 al teration, show a pRb al teration (Yeager et al. 1995).

On the basis of these observations, we hypothesized a new model of TCC pathogenesis in which overcoming cellular senescence by mechanisms that include either $\mathrm{pRb}$ or p16 loss, is a critical step in progression. This model of cancer pathogenesis is based on the hypothesis that senescence is a major tumor suppressor mechanism. In such a model, cells that have bypassed senescence survive to accumulate the multiple mutations required for malignant transformation, whereas cells that senesce cannot progress, regardless of their accumulated mutations. In the current study, we tested this new model of tumorigenesis in which overcoming the senescence block is critical to progression. We examined the status of p16 and pRb in a series of tumors representing both superficial and myoinvasive TCCS. We also examined the status of p53 in these same tumors. Our results show that p53 al terati on is frequently associated with invasive TCC, but correlates best with the phenotype of genome instability, whereas overcoming the senescence bl ock by mechanisms that include p16 or pRb alteration corre 
Overcoming senescence in bladder cancer progression

lates best with progression to myoinvasive cancer. A striking characteristic of all superficial tumors in this study was their ability to show elevated p16 and to senesce spontaneously after a limited number of population doublings.

\section{Results}

Superficial TCCs initially grew in vitro, all showed elevated p16 levels, and senesced spontaneously

Initially, all 13 TCC biopsies (seven superficial tumors and six myoinvasive cancers) grew well in vitro using culture conditions that essentially excluded growth of nonepithelial cell types (Reznikoff et al. 1983). All showed transitional cell epithelial morphology reminiscent of normal HUCs (Fig. 1A), but with several different transformed morphologies. For example, some superficial papillary TCCs formed three-dimensional structures in vitro, reminiscent of their in vivo papillary morphology (Fig. 1B).

All of the superficial TCCs showed a limited lifespan in vitro and spontaneously senesced between P2 and P6. Western blot analysis was used to determine the p16, $\mathrm{pRb}$, and p53 status of these TCC s in the primary culture (P0) and at senescence. Results showed that six superficial TCC s contained relatively low p16 levels at P0, but markedly elevated p16 levels at senescence, similar to
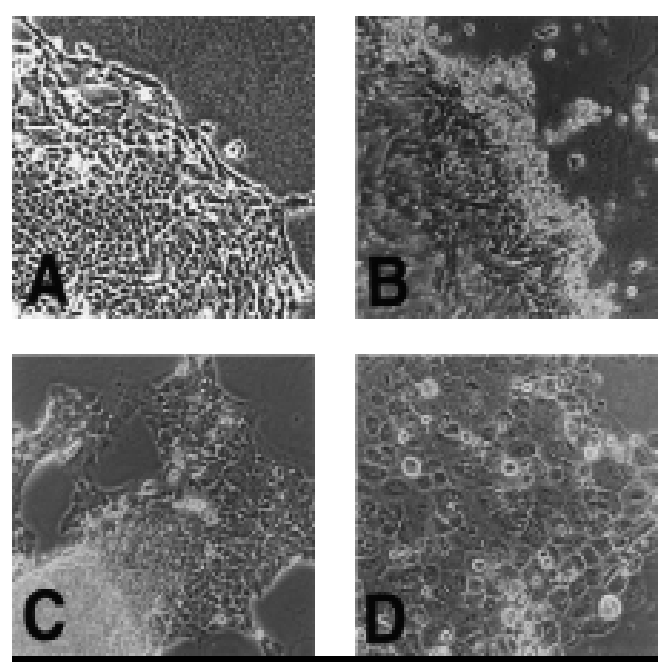

Figure 1. Morphology of TCC compared to HUC in culture. (A) Normal HUCs show typical epithelial morphology with tightly adherent monolayers of polygonal cell. N ote the lack of mesenchymal cell contamination. Original magnification, 200x (B) Superficial papillary TCC s al so show similar epithelial cellular morphology in vitro, but unlike normal HUCs, often form three dimensional structures at the leading edge of growth reminiscent of their in vivo multilayered morphology. Original magnification, 200× (C) M yoinvasive papillary TCC 96-2 also formed a cell line that retained its ability to form three-dimensional structures after prolonged culture. Original magnification, 200x (D) Myoinvasive TCC 96-1 with flat morphology in vitro is shown here. Original magnification, $200 x$

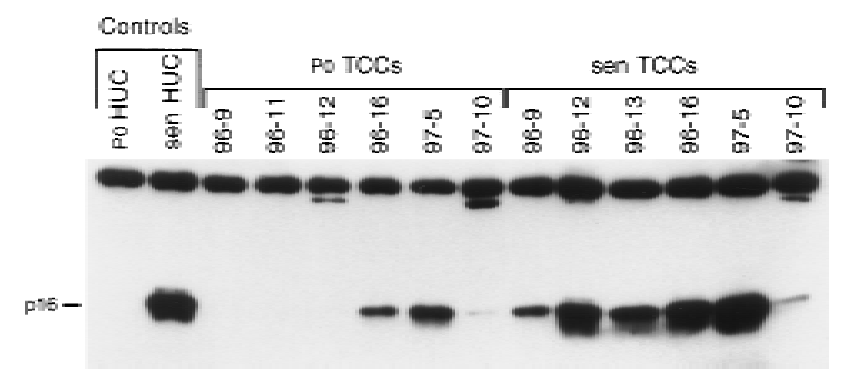

Figure 2. Western blot analysis for $\mathrm{p} 16$ at $\mathrm{P} 0$ and at senescence in superficial TCCs. p16 was el evated in all of the six superficial TCCS at senescence compared to P0, similar to the result obtained with normal HUC. N ote al so that some of the superficial TCCs showed p16 in the PO culture. These superficial TCCs senesced earlier in vitro than TCCs with undetectable p16 at PO.

HUC (Fig. 2; Table 1). One superficial TCC was not tested. Two TCCs that senesced at earlier passages than others, TCC 96-16 and TCC 97-5, had detectable p16 in the primary culture suggesting that the senescence process may have begun in vivo (Fig. 2). Levels of $\mathrm{pRb}$ and p53 in the superficial tumors at both P0 and senescence were similar to normal HUC (summarized in Table 1; data not shown) and therefore, were considered to be wild type. Notably, pRb levels were decreased in both superficial TCCs and in normal HUCs at senescence. In addition to p16 elevation and decreased levels of pRb at senescence, superficial TCCs showed other criteria associated with HUC senescence including cessation of cell proliferation, multinucleated and vacuolated cells, and intense $\beta$-galactosidase staining (Fig. 3).

Myoinvasive TCCs grew in vitro, all showed either p16 or pRb loss, and all bypassed senescence spontaneously

In contrast to superficial TCCs, none of six myoinvasive TCCs in this study underwent the characteristic senescence growth arrest seen in normal HUCs, and none showed $\beta$-galactosidase activity at early or late passage (Table 1). Instead, all six myoinvasive TCCs proliferated continuously in culture without evidence of a growth crisis or a latent period and all spontaneously formed apparently immortal cell lines. Some, but not all, myoinvasive papillary TCCs retained their papillary characteristic in vitro (Fig. 1, cf. C with D). Western blot analysis showed alteration of either p16 or pRb at the earliest passage studied in all of six myoinvasive TCCS (Fig. 4 and Table 1). Notably, three myoinvasive TCCs (TCC 94-10, TCC 96-1, and T CC 97-1) lost p16, whereas three (TCC 92-1, TCC 96-2, and TCC 97-6) lost pRb. $\mathrm{N}$ one lost both p16 and pRb. Interestingly, myoinvasive TCCs with pRb loss showed elevated p16 levels similar to those observed in senescent TCCs and senescent HUCs (Fig. 4). Myoinvasive TCCs that lost pRb or p16 showed this loss at P0 (data not shown), as well as at 
Yeager et al.

Table 1. Association of $\mathrm{p} 16, \mathrm{pRb}$, or $\mathrm{p} 53$ loss with myoinvasive TCC

\begin{tabular}{|c|c|c|c|c|c|c|c|c|c|c|c|c|c|c|}
\hline \multirow{2}{*}{$\begin{array}{l}\text { Sample } \\
\text { code }\end{array}$} & \multirow{2}{*}{\multicolumn{2}{|c|}{ Age/sex }} & \multirow[b]{2}{*}{ Stage/grade } & \multirow{2}{*}{$\begin{array}{c}\text { Crisis/Sen } \\
\text { or Imm }\end{array}$} & \multicolumn{3}{|c|}{$\beta-G a l$} & \multicolumn{2}{|c|}{ p16 } & \multicolumn{2}{|c|}{$\mathrm{pRb}$} & \multicolumn{3}{|c|}{ p53 } \\
\hline & & & & & P0 & Sen & Imm & Sen & Imm & Sen & Imm & PO & Sen & Imm \\
\hline $96-16$ & 52 & M & Ta I & t/Sen & + & + & N.A. & wt & N.A. & wt & N.A. & wt & wt & N.A. \\
\hline $97-10$ & 45 & M & Ta I & t/Sen & - & + & N.A. & wt & N.A. & wt & N.A. & wt & wt & N.A. \\
\hline $96-12$ & 53 & $\mathrm{~F}$ & Ta I & t/Sen & - & + & N.A. & wt & N.A. & wt & N.A. & wt & wt & N.A. \\
\hline $96-13$ & 81 & $\mathrm{~F}$ & Ta I & t/Sen & - & + & N.A. & wt & N.A. & wt & N.A. & N.D. & wt & N.A. \\
\hline $96-9$ & 71 & M & Ta I & t/Sen & - & + & N.A. & wt & N.A. & wt & N.A. & wt & wt & N.A. \\
\hline $97-5$ & 81 & $\mathrm{~F}$ & Ta I/III & t/Sen & + & + & N.A. & wt & N.A. & wt & N.A. & wt & wt & N.A. \\
\hline $96-11$ & 81 & $\mathrm{~F}$ & Ta/T1 I/III & t/Sen & - & + & N.A. & N.D. & N.A. & wt & N.A. & wt & N.D. & N.A. \\
\hline--- & - & - & ---- & --- & - - & - - & - - & -- & - - & - - - & - - & -- & - - & --- \\
\hline $97-1$ & 28 & M & $\mathrm{T} 1 / \mathrm{T} 2 \quad \mathrm{I} / \mathrm{II}$ & $-11 \mathrm{~mm}$ & - & N.A. & - & N.A. & $\mathrm{mu}$ & N.A. & wt & wt & N.A. & wt \\
\hline $94-10$ & 74 & M & T3 II/III & $-11 \mathrm{~mm}$ & N.D. & N.A. & - & N.A. & $\mathrm{mu}$ & N.A. & wt & wt & N.A. & wt \\
\hline $96-1$ & 48 & M & T3 II/III & $-11 \mathrm{~mm}$ & - & N.A. & - & N.A. & $\mathrm{mu}$ & N.A. & wt & N.D. & N.A. & $\mathrm{mu}$ \\
\hline $96-2$ & 67 & M & T3 II/III & $-1 \mathrm{Imm}$ & - & N.A. & - & N.A. & wt & N.A. & $\mathrm{mu}$ & N.D. & N.A. & $\mathrm{mu}$ \\
\hline $97-6$ & 60 & M & T3 III & $-11 \mathrm{~mm}$ & - & N.A. & - & N.A. & wt & N.A. & $\mathrm{mu}$ & $\mathrm{mu}$ & N.A. & $\mathrm{mu}$ \\
\hline 92.1 & 80 & $\mathrm{~F}$ & T4 III & $-1 \mathrm{~mm}$ & N.D. & N.A. & - & N.A. & wt & N.A. & $\mathrm{mu}$ & $\mathrm{mu}$ & N.A. & $\mathrm{mu}$ \\
\hline
\end{tabular}

Shown here are the results of the analysis of TCCs for senescence (Sen) activity as a marker or spontaneous immortal ization (Imm). Also shown are results of western analysis to determine wild-type (wt) vs. mutant (mu) levels of p16, pRb, or p53 in samples of superficial TCCs (shown above the broken line) and myoinvasive TCCs (shown below the line).

later passages (Fig. 4). Thus, the p16 and pRb alterations observed occurred in vivo, not as a result of mutation in vitro. The association between the myoinvasiveness in these TCCs and p16 or pRb alteration was significant $(P=0.001)$, as was the association between myoinvasiveness and bypassing senescence $(P=0.001)$.

Immunohistochemical (IHC) studies were done on al I six myoinvasive TCCs as an additional method to assess the status of $\mathrm{pRb}$. Wild-type $\mathrm{pRb}$, but not mutant $\mathrm{pRb}$, localizes in the nucleus (Cordon-Cardo et al. 1997). pRb was present in the nucleus of the three myoinvasive TCCs that lost p16, but was absent in the nucleus of those that showed pRb loss and el evated p16 (data not shown). Controls, without primary antibody or with an irrel evant antibody, failed to show staining. Thus, IHC results were in agreement with Western blot analysis.

Molecular analyses were performed to identify the mechanisms of CDKN2/p16 inactivation in the three myoinvasive TCCs that did not show p16 by Western blot analysis. Southern blot analysis showed CDKN2/ p16 inactivation by homozygous deletion in two of the
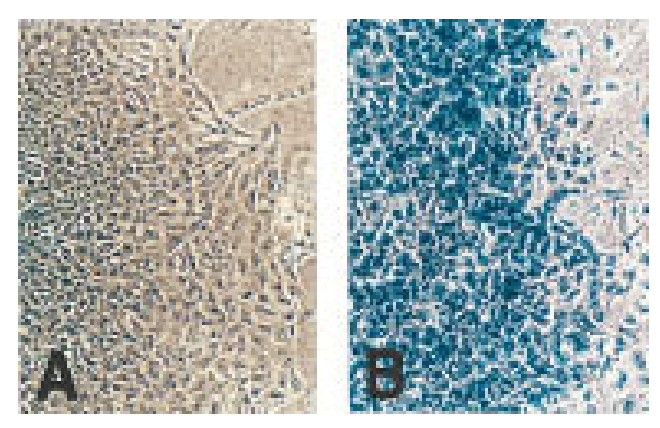

Figure 3. Shown here is an example of staining for $\beta$-galactosidase activity at $\mathrm{PO}$ and at senescence in the same superficial TCC sample TCC 96-12. (A) The proliferating TCC culture shows no $\beta$-galactosidase activity; (B) the same TCC culture, when senescent, shows strong $\beta$-gal actosi dase activity. Original magnification, 100x myoinvasive TCC lines, TCC 96-1 and TCC 97 (Fig. 5). Southern bl ot analysis al so reveal ed hypermethylation of one allele in the third myoinvasive cell line TCC 94-10. CDN A sequencing of TCC 94-10 showed that the second allele was expressed but contained a frameshift mutation in exon 2, codon 7 that caused a premature termination signal (Table 2). Thus, no protein was detected by Western blot analysis.

\section{Some, but not all, myoinvasive TCCs contain TP53 mutations}

To assess the p53 status in myoinvasive TCCs, Western bl ot analysis was done at early and later passages in culture. At the earliest passages studied, five of six myoinvasive TCCs showed alterations in p53 levels, when compared to HUC levels, by Western blot analysis. Four TCCs showed stabilized p53, and a p53 signal was absent in one TCC at both early (data not shown) and later

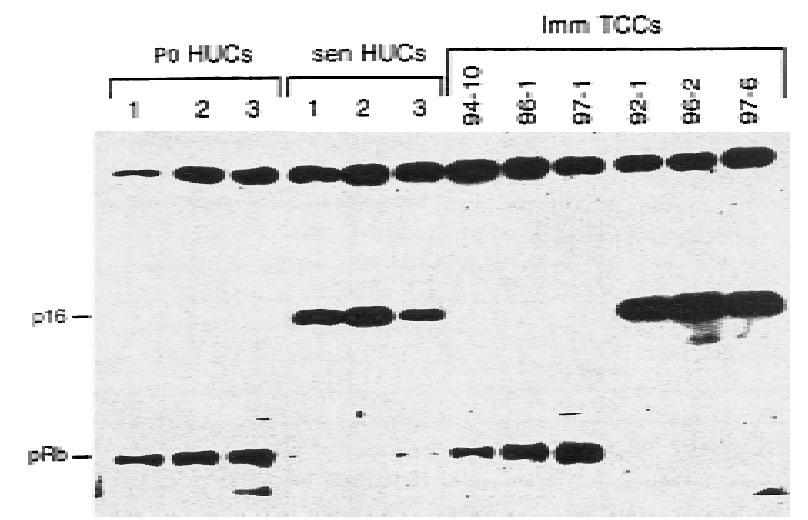

Figure 4. Western blot analysis at approximately P15 for p16 and $\mathrm{pRb}$ in the six myoinvasive TCC cell lines in this study. Three myoinvasive TCCs show no detectable p16, whereas three show no detectable pRb. Results done at P0 show the same results. The nonspecific band is shown to demonstrate protein loading. 


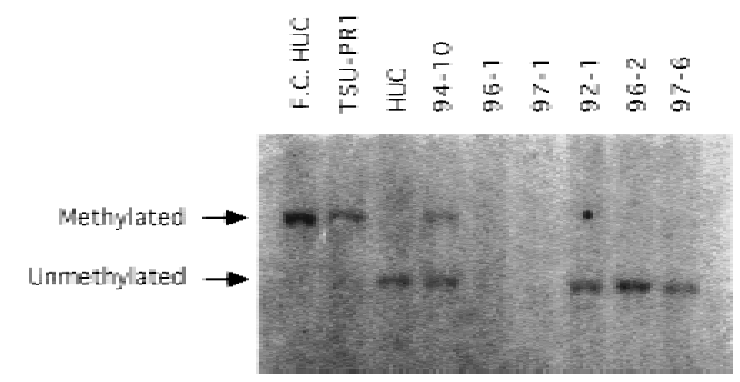

Figure 5. Southern bl ot analysis for CDKN2/p16 methylation. Flanking cut (F.C.) HUC was digested only with the enzyme for the F.C. EcoRI. All other samples were digested with both EcoRI and Sacll. TSU-PR1 is a prostate cell line containing methylated CDKN2/p16 that was used as a positive control for methylation. The three TCC cell lines that expressed elevated p16 showed no altered methylation. In contrast, the three TCCs that failed to show p16 by Western blot analysis showed genetic alterations by Southern blot analysis. TCC 96-1 and TCC 97-1 both contained homozygous CDKN 2/ p16 del etions. TCC 94-10 had one methylated allele. Sequencing of cDN A showed a mutation in the second allele as discussed in text.

passages (Fig. 6). To further investigate the underlying mechanisms for the altered p53 levels observed using Western blot analysis, TP53 was sequenced in all six myoinvasive TCC lines. Two of the six myoinvasive TCCs had wild-type TP53 by sequencing, including one line, TCC $97-1$, that showed stabilized p53 by Western blot analysis (Fig. 6; Table 2). Two of the TCCs with stabilized p53 contained a single TP53 mutation, whereas one TCC, TCC 92-1 (the only TCC that showed nodal metastases in the patient), contained five different mutations affecting 4 exons (Fig. 6; Table 2). N otably, TCC 96-2, the TCC in which p53 was not detected using Western bl ot analysis, contained a splice mutation consistent with p53 absence (Table 2). The association between a mutant TP53 and myoinvasive TCC was determined to have $P=0.04$ using Fisher's test.

\section{Chromosome instability associates with TP53} mutation

To assess and compare chromosome stability in superficial and myoinvasive TCCs, CGH was preformed on 12 of 13 TCCs in this study (T able 3). Results showed few or no chromosome losses or gains in the superficial TCCs or in the myoinvasive TCCs with wild-type TP53. For example, no changes were detected in two superficial TCCs, two had two changes, one had three changes, and one had four changes. The two myoinvasive TCCs with wild-type TP53 each contained two alterations. In contrast, the four myoinvasive TCCs containing TP53 mutations had 13,20, 23, and 32 changes, respectively, and all were aneuploid (Table 3). The association between TP53 mutation and chromosome instability was significant $(P=0.001)$.

Nonrandom chromosome alterations associated with TCC progression

CGH was also used to identify potentially important chromosomal losses and gains associated with TCC progression and bypassing of senescence. Although many of the chromosome alterations observed in myoinvasive TCCs were apparently random changes resulting from genome instability, some alterations appeared to be nonrandom (Table 3). For example, five of six myoinvasive TCCs lost the 8p22-pter region (four of six lost 8p21pter), five gained 20q11-q12, four gained $5 p$, and four gained 7p (Fig. 7A,B). In addition, all four of the superficial TCCs in which chromosome alterations were detected by CGH showed loss of the 9q chromosome arm. Three myoinvasive TCCs al so lost 3p13-p14.

A simple stochastic statistical model was used to assess whether or not such patterns (Fig. 7A,B) of al teration were consistent with sporadic change. Separate analyses of losses and gains showed that they were not related to sporadic change, and that certain regions exhibited higher than expected rates of change. Global $P$ values against the sporadic null hypothesis were $0.001,0.005$, and 0.001 for late stage gain, late stage loss, and early stage changes, respectively. Under the alternative hypothesis that certain regions exhibit change at higher rates than other regions, we calculated the probability that each arm had an el evated change. Elevated changes of $+20 q 11-q 12(0.99)$ and $-8 p 22-p t e r(0.94)$ occurred in myoinvasive TCC and of $-9 q(0.994)$ in superficial TCC (Fig. 7C,D). The probabilities that $5 p+$ and $7 p+$ were al so

Table 2. CDKN 2 and TP53 mutations in myoinvasive TCCS

\begin{tabular}{lll}
\hline Sample code & \multicolumn{1}{c}{ CDKN2/p16 } & \multicolumn{1}{c}{ TP53 } \\
\hline $97-1$ & homozygous deletion & wild type \\
$94-10$ & methylation and FS (exon 2, codon 74) & Wild type \\
$96-1$ & homozygous deletion & mutant (exon 5, codon 175) \\
$96-2$ & Wild type & Mutant (exon 7, codon 261, SM) \\
$97-6$ & Wild type & Mutant (exon 5, codon 162) \\
$92-1$ & Wild type & mutant (exon 5, codon 158) \\
& & (exon 6, codon 224) \\
& & (exon 7, codon 228) \\
& & (exon 8, codons 280 and 294) \\
\hline
\end{tabular}

(FS) Frameshift mutation; (SM ) splice mutation. CDKN 2 al terations were detected by Southern anal ysis and genomic sequencing; TP53 alterations were detected by genomic sequencing. 


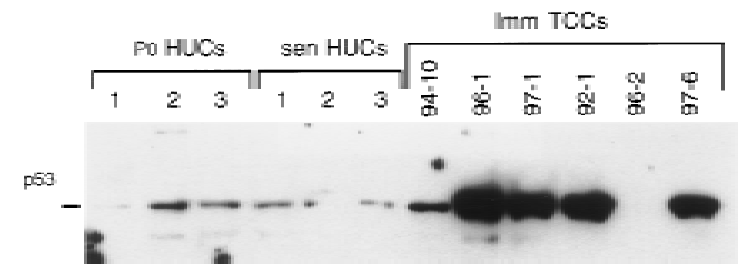

Figure 6. Western blot analysis of myoinvasive TCCs for p53. $\mathrm{N}$ ote relatively low, but detectable, p53 levels at P0 and in senescent HUCs and in one myoinvasi ve TCC. In contrast, note altered p53 levels in five myoinvasive TCCs, four showing stabilized p53 and one TCC lacking a detectable p53 signal. N otably, one of the TCC with stabilized p53, TCC 97-1, had wildtype TP53 by sequencing. Results of later passage cultures are shown, but results were the same at P0. Thus, no changes occurred in culture.

elevated in myoinvasive cancers were calculated using the same method and were significant at 0.87 and 0.83 , respectively. In this small study, the three losses of 3p13-p14 was just below significance.

\section{Discussion}

Previously our laboratory demonstrated that p16 is elevated in cultured normal HUCs at senescence (Reznikoff et al. 1996b) and that HUC immortal ization in vitro is essentially al ways associated with either p16 or pRb alteration (Yeager et al. 1995). These observations sug- gested an important biological role for p16 in limiting normal cell proliferation and led us to hypothesize that overcoming cellular senescence might be an important step in cancer pathogenesis in vivo, as well as in immortalization of this cell type in vitro. N otably, our observation that p16 el evation occurs at senescence in human epithelial cells has been confirmed (Serrano et al. 1997). The many reports showing p16 (or pRb) alterations in clinical bladder tumors (for review, see Reznikoff et al. 1996a) are consistent with our hypothesis that overcoming cellular senescence is critical to bladder cancer pathogenesis in vivo.

In the present study, we specifically tested the hypothesis that myoinvasive TCCs, but not superficial TCCs, have bypassed the $G_{1}$ p16-mediated senescence growth arrest block. Senescence is defined as programmed cell death that occurs after a limited number of population doublings. Senescence occurs in vitro (Hayflick 1974) and in vivo (Dimri and Campisi 1994). We al so tested the hypothesis that myoinvasive TCCs, but not superficial TCCs, would contain a genetic al teration, such as p16 or pRb loss, consistent with bypassing senescence in this cell type. The results supported both hypotheses, both at a $P$ value of 0.001 . All the myoinvasive TCCs bypassed senescence and spontaneously formed cell lines and all contained either a p16 or a pRb al teration. In contrast to myoinvasive TCCs, the superficial TCCs, like normal HUCs, underwent cellular senescence after a limited lifespan in vitro and this was al ways associated with wild-type $\mathrm{pRb}$ and the expected el evated p16 levels. In-

Table 3. Association of p53 alteration with genome instability

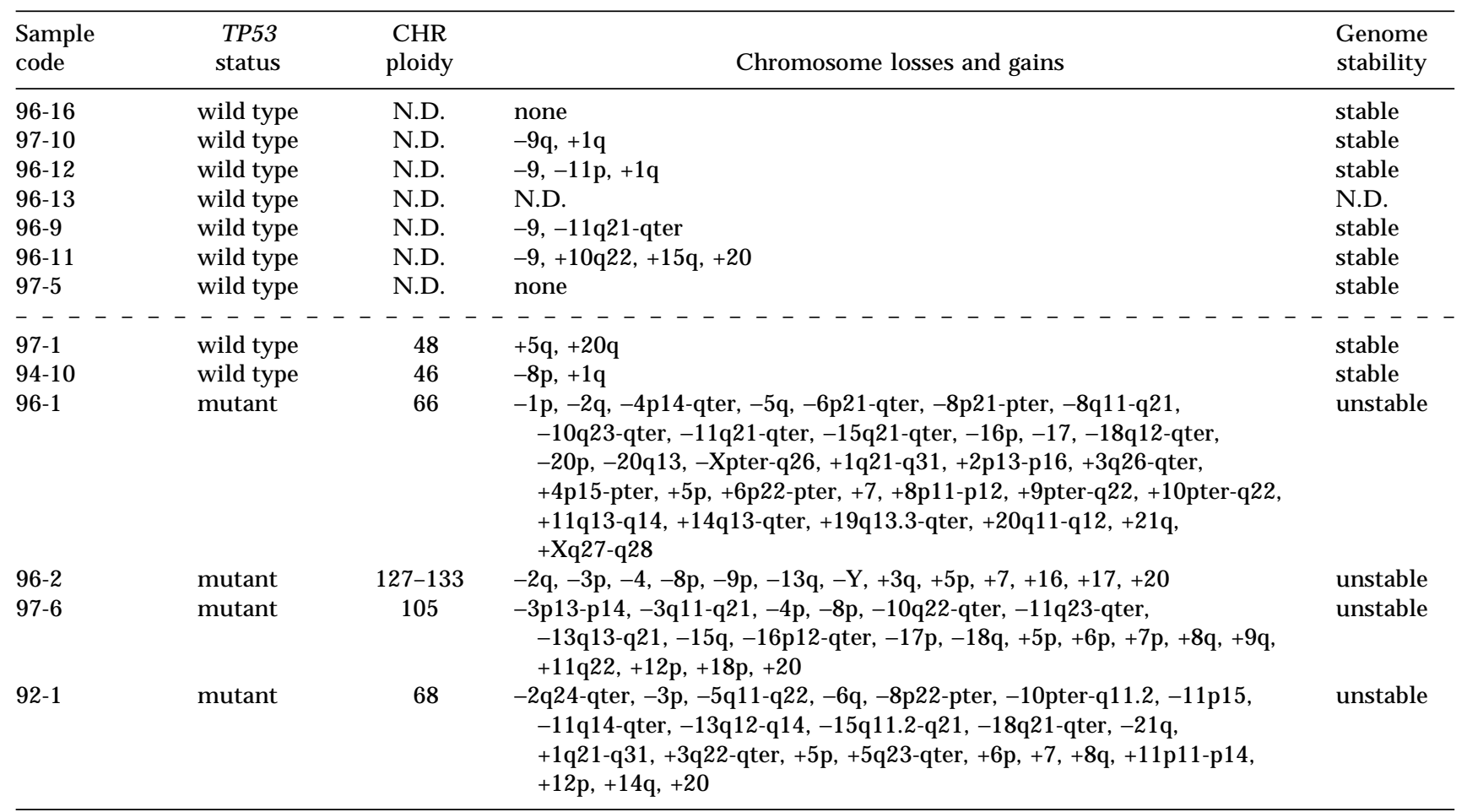

Determination of whether chromosome regions were lost or gained was done using CGH. Ploidy was determined by karyotypic analysis. The tumors above the broken line were superficial, and the tumors below the line were muscle invasive. 
Late stage losses
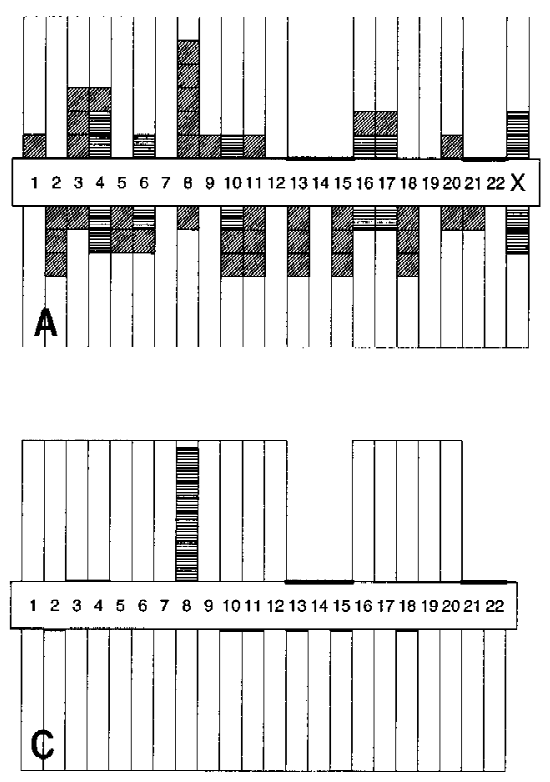

Late stage gains
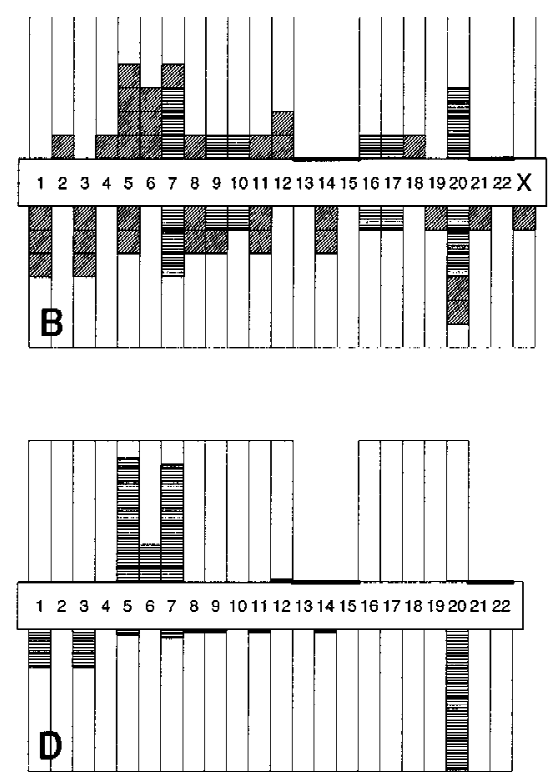

Figure 7. Chromosome arm losses and gains in myoinvasive TCCs as detected by $\mathrm{CGH}$. (A,B) Chromograms recording the number of six myoinvasive TCCs exhibiting a change on each of 39 nonacrocentric chromosome arms. Horizontal hash marks indicate that the loss or gain on both arms was linked. Diagonal marks indicate that the loss or gain was not linked to the other chromosomal arms. (C,D) Probabilities calculated under the simple stochastic model conditional on the chromograms. The arm has an elevated rate of loss or gain. $(A, C)$ Late0stage losses; $(B, D)$ late-stage gains. Results of cal culations are presented in the text. terestingly, the wild-type $\mathrm{pRb}$ levels were lower at senescence in superficial TCCs, as they are in normal HUCS at senescence (Reznikoff et al. 1994). N either the mechanism by which p16 is elevated at senescence nor the mechanism by which $\mathrm{pRb}$ is decreased at senescence are currently known. The association between senescence and superficial TCC was also significant $(P=0.001)$. These data demonstrate for the first time in any system that superficial tumors retain the ability to senesce. This is also the first report to our knowledge specifically associating the phenotype of tumors bypassing senescence with a progressed cancer phenotype in vivo. The ability to demonstrate this difference was dependent on the establishment in our laboratory of culture techniques that support growth and immortalization of superficial and invasive human bladder tumor cells in vitro.

These data are significant for several reasons. First, they support a new model of epithelial cell tumorigenesis in which normal cellular senescence mediated by p16 accumulation can be considered an important tumor-suppressor mechanism that must be overcome for the development of the fully neoplastic phenotype. These data also suggest that alterations in at least two different genes, namely CDKN2/p16 or RB, can fulfill the same requirement (in this case overcoming the $G_{1}$ senescence block) in multistep tumorigenesis. Amplification of CCND1, the gene for cyclin D, may also provide a less frequently used mechanism for bypassing senescence. In support of this, recent studies have identified a small percent of bladder tumors in which cyclin D1 is overexpressed (Ohta et al. 1997; Shin et al. 1997). Thus, different genetic alterations may achi eve the same biological end point in tumorigenesis. This finding is consistent with the genetic heterogeneity seen in tumors of the same types, including TCCs. This new model is attractive because it is consistent with the requirements for continued proliferation to accumulate and fix genetic alterations in multistage tumorigenesis (Reznikoff et al. 1996a). The presence of $\mathrm{p} 16$ or pRb alterations in many tumor types other than TCCs suggests that our new model may apply broadly in the pathogenesis of human cancer (Cairns et al. 1994, 1995; Balazs et al. 1997; Lowe and Jacks 1997).

A number of studies have implicated tel omere shortening as a critical factor in the onset of senescence (for review, see Harley 1995). Therefore, in another study we also examined both telomerase activity and telomere lengths in normal HUCs and in superficial and invasive TCCs (Belair et al. 1997). We found that uncultured tissue biopsies of normal bladder urothelium were negative, whereas all uncultured TCC biopsies (both superficial and invasive) were positive. However, we al so discovered that the same normal uroepithelium, when established as proliferating cultures showed telomerase activity (albeit somewhat lower than in the TCC samples), as did prol iferating normal human prostate and mammary epithelium. Furthermore, we demonstrate that normal urothelium with telomerase activity does not show telomere shortening at senescence. Thus, we concluded that tel omerase activity is a biomarker of proliferation, not of malignant transformation (Belair et al. 1997). Relevant to the present study, two chromosome regi ons encoding the RN A component of telomerase and the active component, namely $3 q 26$ and 5 p14, respectively (M eyerson et al. 1997; Soder et al. 1997), were both found to be overrepresented in the TCC lines in this study (Table 3). The $5 p$ arm was gained in five invasive TCCs and was likely to be si gnificant at 0.87 . Only three TCCs gained $3 q$, but significantly the smallest regions 
gained was 3q26-qter. Thus, it is reasonable to hypothesize that these gains may contribute to the higher level of telomerase activity observed in these TCCs in our previous study (Belair et al. 1997).

These data give other insights into the pathogenesis of superficial bladder cancers. The loss of a whole chromosome 9 is the most frequent genetic alteration in TCCs and is grade and stage independent, suggesting that a chromosome 9 loss is an early event (Ross et al. 1996). Because CDKN2/p16 is located on 9p21, CDKN2/p16 might be considered a candidate tumor-suppressor gene for early stage TCC. Our data do not support that hypothesis. One superficial TCC lost $9 q$ and three lost the entire chromosome 9 making $9 q$, not $9 p$, the common regi on lost. Furthermore, none of the superficial TCC s in our study showed p16 alteration. Therefore, we would support a model in which loss of a gene (not yet identified) on $9 q$ is an important early event in TCC pathogenesis. However, these data do support a model in which p16 loss (or pRb loss) is important in TCC progression and would be a poor prognostic indicator if present in a superficial TCC. We and others do not find that p16 alteration occurs early in TCC pathogenesis (Okajima et al. 1996). One study, however, using fluorescent in situ hybridization (FISH) with CDKN2/p16 probes shows that p16 al teration can be detected in uncultured superficial TCCs (Balazs et al. 1997). However, in the latter study, the TCCs were not examined for other changes required for overcoming senescence and were not cultured to determine whether they had a limited lifespan. Thus, the rel evance of those findings to our study is uncertain.

Previously we reported that immortalization of human papillomavirus-transformed HUCs was always accompanied by chromosome losses and gains, in addition to either pRb or p16 loss (Y eager et al. 1995; Reznikoff et al. 1996b). The most frequent gains were $+20 q 11-q t e r$ and $+5 p$ and the most frequent loss was $-3 p 13-p 14.2$ (Savelieva et al. 1997; Vieten et al. 1997). It is remarkable that these same regions were altered in the myoinvasive TCCs that had overcome senescence in this study. For example, there are three examples of $3 p$ loss in myoinvasive TCCs in this study and the smallest common region lost, 3p13-p14, was precisely the same common region lost in HUCs transformed in vitro (Vieten et al. 1997) and identified in uncultured clinical TCCs (Kallioniemi et al. 1995). In addition, loss of 8p was highly significant in the current study. Three TCCs showed -8p21, a region lost in many human cancers, including bladder cancers (Knowles et al. 1996). Our study now associates 8p21 loss for the first time with overcoming cellular senescence and again with myoinvasive late stage cancers. $\mathrm{N}$ otably, $3 p$ and $8 p$ I osses have been associated with late stage cancers by other investi gators (Hubuchi et al. 1993; Knowles et al. 1994), which is consistent with our new model. Finally, we were the first laboratory to associate gain of 20q11-qter genes with immortalization (Reznikoff et al. 1994). We al so reported a strong association between amplification of 20q13.2 and genomic instability (Savelieva et al. 1997). The cur- rent study extends these findings by better defining the regi on associated with immortalization, as the smallest common region gained in a myoinvasive TCC was $20 q 11-q 12$. Because gain of $20 q$ is found in many human cancers, these finding have value for understanding its significance. In summary, our new data support a role for losses of 3p13-p14, 8p22-pter or 8p21-pter and gain of $20 q 11-q 12$ in bypassing senescence and support our hypothesis that loss of p16 or pRb alone is insufficient for immortalization. Other potential changes in chromosome balance in TCCs in this study not discussed above include +1q31-qter, +6p22-pter, -4p14-pcen, -10q22qter, and -18q21-qter. Alterations in these regions are reported in other human cancer types (Mertens et al. 1997).

Previous studies reported that T CC cell lines showed a higher percentage $(54 \%)$ of p16 alterations than uncultured TCC biopsies (19\%). Thus, it was proposed that p16 mutations occurred in vitro (Spruck et al. 1994b). Our data would support an alternative explanation (Cairns et al. 1994), namely that CDKN2/p16 mutations are more frequent in TCC cell lines because TCCs with p16 alterations form cell lines more frequently. In our study, the kinetics of cell line formation in vitro by myoinvasive TCCS, namely continuous growth of TCC without a latent period or crisis, are inconsistent with a model in which CDKN2/p16 mutations occurred in culture. In addition, the failure of any of the superficial TCCs (cultured under the same conditions) to form cell lines argues against random CDKN2/p16 mutations in vitro as an explanation for our observations. Interestingly, our data illustrate the futility of using Western bl ot analysis to assess CDKN2/p16 status in uncultured TCC biopsies, as wild-type p16 is low to undetectable using this method until cells approach senescence. Finally, a recent observation in our laboratory illustrates that a myoinvasive component of a heterogeneous TCC can be detected in our system because of its ability to adapt to culture (data not shown).

Clearly, our studies demonstrate that the use of a single marker, including ei ther p53 or pRb, is inadequate for either prognosis or classification of TCCS. In addition, our studies al so demonstrate that using pRb or p53 alterations together, although better than singly, as prognostic markers or to identify progressed cancers (CordonCardo et al. 1997) would miss myoinvasive TCCs that evolve through p16 alteration or progress without p53 alteration. For example, three myoinvasive TCCs in this study had both p53 and pRb alteration, but two had neither alteration. We would propose that p16 and pRb alteration represent two mechanisms in the same $\mathrm{p} 16 / \mathrm{pRb}$ pathway to bypass the senescence block. Furthermore, our study suggests caution in using IHC as a primary means to detect TP53 mutations as previously noted (Lowe and Jacks 1997). One myoinvasive TCC (i.e., TCC 97-1) showed stabilized p53 but TP53 was wild type by sequencing, whereas one myoinvasive TCC with mutated TP53 would not have been identified as altered as it lacked detectable p53 protein (TCC 96-2). N either TCC would be classified accurately by IHC that uses stabi- 
lized p53 as an end point for mutated TP53. We propose using a phenotype as a prognostic marker and allowing for different combinations of genetic al terations to give rise to that same phenotype in tumorigenesis.

In summary, the present work supports a new model of human tumorigenesis in which overcoming cellular senescence is a critical step. The present work also defines a role for p16 or pRb alteration, 20q11-q12 gain, and 8p21-pter or 3p13-p14 loss in overcoming cellular senescence. The frequency of $\mathrm{p} 16$ and $\mathrm{pRb}$ al terations, as well as of $20 q$ gains and $8 p$ losses in many human cancer types suggests that this new model may apply broadly in the pathogenesis of human carcinomas.

\section{Materials and methods}

\section{TCC biopsies}

Thirteen biopsies of bladder tumors were obtained from 13 different patients (five women and eight men) after transurethral resection of the bladder or cystectomy at the University of Wisconsin Hospital and Clinics (Table 1). Tumors were immediately placed into growth medium (see below). A representative piece of each biopsy was sent for pathological evaluation. Pathol ogy slides were read independently by two pathologists. All but three tumors in this series were papillary, and all were classified as TCCs. The samples ranged from Ta to T4 (TN M classification) and from grade I to III. All the superficial tumors were primary papillary tumors. Only three of the myoinvasive tumors were papillary (TCC 97-1, TCC 94-10, and TCC 96-2). Two of the myoinvasive tumors (TCC $94-10$ and TCC 96-1) were recurrences. One patient had extensive nodal metastases (TCC 92-1). Medical histories revealed that 11 of 13 bladder cancer patients were smokers.

\section{TCC cell culture}

Tumor biopsies were set up for P0 cell culture using an explant technique developed for TCC (Reznikoff et al. 1983). Briefly, specimens were placed into a sterile glass Petri dish containing culture medium and then cut up into pieces, $\sim 1 \mathrm{~mm}^{2}$ in size, using sterile forceps and scal pel. The explants were then transferred to 100-mm Petri dishes coated with collagen that had been preequilibrated with growth medium (Reznikoff et al. 1987). The growth medium, 1\% FBS-F12+, consisted of Ham's F12 (GIBCO, Grand Island, NY) containing 1\% fetal bovine serum (FBS; GIBCO) and growth supplements optimized for human uroepithelial cells (Reznikoff et al. 1987). Initially, $2 \mathrm{ml}$ of medium was added. An additional 2-3 $\mathrm{ml}$ was added after $24 \mathrm{hr}$. Then, the $7 \mathrm{ml}$ of medium was used and replaced three times a week. After 7-10 days, or when the cultures were $80 \%$ confluence, the explants were removed and the epithelial cells were dispersed for passage using $0.1 \%$ EDTA. Cells from multiple P0 dishes initiated from a single TCC sample were pooled. From this pool, aliquots were taken for DNA and protein extraction and for $\beta$-galactosidase activity, as described below. The re maining cells were replated and serially cultured to determine whether they senesced or spontaneously immortalized. Senescence occurred between P2 and P6. In this study TCCs that failed to senesce, spontaneously formed cell lines. HUCs, used as normal controls, were cultured exactly as previously described (Reznikoff et al. 1987).

\section{Assessment of $\beta$-galactosidase activity}

$\beta$-Galactosidase activity was used as a marker for cellular se- nescence (Dimri et al. 1995). TCC were first tested at early passage, usually $\mathrm{PO}$, at senescence, or after senescence if cells did not senesce. The $\beta$-galactosidase assay was done as described previously (Dimri et al. 1995). Briefly, all cells were fixed with formalin:glutaral dehyde, rinsed, and then incubated in SA- $\beta$-galactose stain solution for $18 \mathrm{hr}$. Cells were then rinsed with PBS. Senescent cells showing $\beta$-gal actosi dase activity were identified by their blue color using a microscope.

\section{Western blot analysis}

Western blot analysis was used for initial assessment of p16, $\mathrm{p} 53$, and pRb status and was performed as described previously (Yeager et al. 1995). Briefly, cells were lysed in ECB buffer [50 $\mathrm{mm}$ Tris- $\mathrm{Hcl}$ at pH 8.0; $120 \mathrm{~mm} \mathrm{NaCl} ; 100 \mathrm{~mm} \mathrm{NaF} ; 0.2 \mathrm{~mm}$ $\mathrm{Na}_{2} \mathrm{VO}_{4} ; 0.5 \% \mathrm{~N} \mathrm{P}-40$ with $10 \mathrm{mg} / \mathrm{ml}$ each of leupeptin, PM SF, and aprotinin (Sigma, St. Louis, MO)]containing protease inhi bitors, proteins were quantified by Bradford assay, and then loaded ( $50 \mu \mathrm{g} /$ lane), and run on a $12 \%$ SDS-polyacrylamide gel. Proteins were transferred to a nitrocellulose membrane and checked for equal loading by Ponseau S staining (Sigma, St. Louis, MO). The test antibody was added next and immunoreactive proteins were detected by enhanced chemiluminescence (Pierce, Rockford, IL). Monoclonal antibodies to pRb (14001A, PharM ingen, San Diego, CA) and p53 (AB-2, Oncogene Research Products, Cambridge, MA) were used. A polyclonal p16 antibody (C-20, Santa Cruz Biotechnology, Santa Cruz, CA) was used. All Western blot analyses were repeated at least twice.

\section{IHC Studies}

IHC was used as a second assay to assess loss of wild-type pRb. Cells were grown on chambered glass slides (N unc, Naperville, IL) and fixed with glutaraldehyde and formalin (Dimri et al. 1995). The pRb monoclonal antibody (14001A Pharmingen) was used at a 1:1000 dilution to detect wild-type nuclear $\mathrm{pRb}$ using the protocol of the Histomark Streptavidin-HRP system (Kirkegard and Perry Laboratories, Gaithersburg, MD). TrueBlue peroxidase substrate (Kirkegard and Perry) was used for final detection. Groups without primary $\mathrm{pRb}$ antibody and with an irrelevant anti-SV40 T antigen antibody (Oncogene Research Products) were used as controls. IHC was done at least twice for all samples.

\section{Southern blot analysis}

Southern blot analysis was used to detect CDKN2/p16 deletions or altered methylation in the six myoinvasive TCC samples that formed cell lines. DNA was isolated using the guanidine isothiocynate method (Savelieva et al. 1997). Southern blot analysis for CDKN2/p16 methylation was done using $10 \mu \mathrm{g}$ of DN A per lane after digestion with both EcoRI and the methylation-sensitive Sacll restriction enzymes (Promega, Madison, WI). DNA was electrophoresed on a $1 \%$ agarose gel and transferred to Hybond $\mathrm{N}+$ membrane (Amersham Life Science, Arlington Heights, IL). The CDKN2/p16 probe, which overlaps the CpG islands of the promoter and exon 1 of CDKN2/p16 was labeled with $\left[\alpha^{-32} \mathrm{P}\right] \mathrm{dCTP}$ using the Rediprime labeling kit (Amersham Life Sciences) and detected using a Phosphorlmager. M ethylation in this region using this method and probe has been correl ated with loss of CDKN 2/p16 expression (M erlo et al. 1995). The human prostate carcinoma cell line TSU-PR 1 was used as a positive control for methylation in this assay. 


\section{DNA sequencing}

Sequencing of TP53 and CDKN2/p16 was done to identify mutations in cells with altered $\mathrm{p} 53$ or p16 by Western bl ot analysis. Genomic sequencing of TP53 was done by Oncor Med (Gaithersburg, MD) according to their published protocol. Briefly, the entire sequences of exons 5 through 9 , as well as 10-20 bases $5^{\prime}$ and $3^{\prime}$ of each exon, were sequenced and compared to wildtype TP53 (GenBank). The same methodology was used in sequencing exons one and two of CDKN2/p16.

\section{$\mathrm{CGH}$}

CGH was used to detect chromosomal losses and gains. Hybridization of differentially labeled tumor and normal DNA to normal metaphase chromosomes was performed exactly as described previously (Kallioniemi et al. 1995). Tumor DNA was labeled with fluorescein-dUTP and normal DNA was labeled with Texas red-dUTP. Both were hybridized to normal metaphase spreads, which were then DAPI stained. Three-color images (fluorescein, Texas red, DAPI) were collected from five metaphase cells for each sample. Green and red fluorescence signals from pter to qter and the ratios of green to red intensity were cal culated for each chromosome in the metaphase spread. In each metaphase cell the absolute fluorescence intensities were normalized so that the average green to red ratio of all chromosomes in the metaphase was one. The final results were expressed as the mean green to red ratio profiles +1 standard deviation, determined from two to three metaphase cells (four observations per chromosome). Loss shown in Table 3 were present in the majority $(>50 \%)$ of cells.

\section{Cytogenetic analysis}

Karyotypic analysis was done using publ ished techniques (Save lieva et al. 1997) to determine the modal chromosome number of each new TCC cell line, as an indicator of genome stability. Briefly, cells were arrested in metaphase by adding $0.2 \mu \mathrm{g} / \mathrm{ml}$ colcemid (GIBCO) for $3 \mathrm{hr}$. Afterward, cells were collected and incubated at $37^{\circ} \mathrm{C}$ with hypotonic solution $(75 \mathrm{~mm} \mathrm{KCl})$. Cells were fixed with 3:1 methanol/acetic acid. Chromosome spreading was done on a humidified slide warmer. Then, cells were trypsin banded and stained with Giemsa. The modal number was determined by counting at least 20 metaphase spreads for each sample.

\section{Statistical methods}

Fisher's exact test was used for two-factor comparisons in stage versus $\mathrm{p} 16 / \mathrm{pRb}$ al teration, stage versus senescence, stage versus p53 alteration, and genomic instability versus p53 alteration. Chromosomal losses and gains measured by CGH (Table 3) were analyzed separately using a modification of the stochastic model developed in earlier work (N ewton et al. 1994). First, a likelihood ratio statistic was cal culated to test the null hypothesis that changes were completely sporadic, versus the alternative hypothesis that certain regions have elevated rates of ge netic change. The likel ihood ratio test was cal ibrated by a parametric bootstrap using $10^{3}-1$-simulated chromograms (Efron and Tibshirani 1993). Second, for each arm we calculated the conditional probability of having an el evated change rate given the observed data and estimated model parameters (Fig. 7). Briefly, the model views changes as stochastic and the presence or absence of an elevated change rate on each nonacrocentric chromosome arm as the outcome of a biased coin toss. Whether or not change is observed on one arm in a tumor is further viewed as the outcome of a biased coin toss, with a higher change probability in the arms deemed to have higher rates. In addition, the model accounts for linked changes (e.g., loss of entire chromosome), by having another biased coin to indicate whether or not such linkage will occur. The modification from N ewton et al. (1994) is that linkage probability is fixed for all chromosomes, reducing the number of parameters and simplifying the calculation.

\section{Acknowledgments}

The publication costs of this article were defrayed in part by payment of page charges. This article must therefore be hereby marked "advertisement" in accordance with 18 USC section 1734 solely to indicate this fact.

\section{References}

American Cancer Society. 1997. Cancer facts and figures1996, 96-300M-No. 5008.96. American Cancer Society, Bethesda, MD.

Bal azs, M., P. Carroll, R. Kerschmann, S. Sauter, and F.M. Waldman. 1997. Frequent homozygous deletion of cyclin-dependent kinase inhibitor 2 (MTS1, p16) in superficial bladder cancer detected by fluorescence in situ hybridization. Genes, Chrom. Cancer 19: 84-89.

Bane, B.L., J.Y. Rao, and G.P. Hemstreet. 1996. Pathology and staging of bladder cancer. Semin. Oncol. 23: 546-570.

Belair, C.D., T.R. Y eager, P.M. Lopez, and C.A. Reznikoff. 1997. Telomerase activity: A biomarker for proliferation-not malignant transformation. Proc. Natl. Acad. Sci. (in press).

Cairns, P., L. Mao, A. Merlo, D.J. Lee, D. Schwab, Y. Eby, K. Tokino, P. van der Reit, J.E. Blaugrund, and D. Sidransky. 1994. Rates of p16 (MTS1) mutations in primary tumors with 9p loss. Science 265: 415-416.

Cairns, P., T.J. Polascik, Y. Eby, K. Tokino, J. Califorino, A. Merlo, L. Mao, J. Hearath, R. Jenkins, W. Westra, J.L. Rutter, A. Bukler, E. Gabrielson, M. Tockman, K.R. Cho, L. Hendrick, G.S. Bova, W. Isaacs, W. Koch, D. Schwab, and D. Sidransky. 1995. Frequency of homozygous deletion at p16/ CDKN 2 in primary human tumors. Nature Genet. 11: 210212.

Catal ona, W.J. 1992. Urothelial tumors of the urinary tract. In Campbell's U rology (ed. P.C. Walsh, A.B. Retik, T.A. Stamey, and E.D. Vaughn), Chapter 28, pp. 1094-1158. W.B. Sanders, Philadel phia, PA.

Cordon-Cardo, C., Z.-F. Zhang, G. Dalbangi, M. Drobnjak, E. Charytonowicz, S.-X. Hu, H.-J. Xu, V.E. Reuter, and W.F. Benedict. 1997. Cooperative effects of p53 and pRb al teration in primary superficial bladder tumors. Cancer Res. 57: 12171221.

Dimri, G.P. and J. Campisi. 1994. Molecular and cellular biology of replicative senescence. Cold Spring Harbor Symp. Quant. Biol. 59: 67-73.

Dimri, G.P., X. Lee, G. Basile, M. Ascota, G. Scott, C. Roskelley, E.E. Medranos, L. Linskens, O. Peoria-Smith, M. Peacocke, and J. Campisi. 1995. A biomarker that identifies senescent human cells in culture and in aging skin in vivo. Proc. Natl. Acad. Sci. 92: 9363-9367.

Efron, B. and R. Tibshirani. 1993. An introduction to the bootstrap. Chapman and Hall, London, UK.

Esrig, D., D. Elmnain, S. Groshen, J.A. Freeman, J.P. Stein, S.-C. Chen, P.W. Nichols, D.G. Skinner, P.A. Jones, and R.J. Cote. 1994. Accumulation of nuclear p53 and tumor progression in bladder cancer. N. Engl. J. Med. 331: 1259-1264. 
Gonzalez-Zulueta, M., C.H. Bender, A.S. Yang, T. N guyen, R.W. Beart, J.M. Van Tornout, and P.A. Jones. 1995. M ethylation of the $5^{\prime} \mathrm{CpG}$ island of the p16/CDKN 2 tumor suppressor gene in normal and transformed human tissues correlates with gene silencing. Cancer Res. 55: 4531-4535.

Harley, C.B. 1995. Telomeres and aging. In Telomeres (ed. E.H. Blackburn and C.W Greider), pp. 247-263. Cold Spring Harbor Laboratory Press, Cold Spring Harbor, NY.

Hayflick, L. 1974. Thelongevity of cultured human cells. Assoc. Am. Geriatr. Soc. 22L: 1-12.

Hollstein, M., D. Sidransky, B. Vogelstein, and C.C. Harris. 1991. p53 mutations in human cancers. Science 253: 49-53.

Hubuchi, T., O. Ogawa, Y. Kakehi, K. Ogura, M. Koshiba, S. Hamazaki, R. Takahashi, T. Sugiyama, and O. Yoshida. 1993. Accumulated allelic losses in the devel opment of urothelial cancer. Int. J. Cancer 53: 579-584.

Kallioniemi, A., O.-P. Kallioniemi, G. Citro, G. Sauter, S. DeVries, R. Kerschmann, P. Caroll, and F. Waldman. 1995. Identification of gains and losses of DNA sequences in primary bladder cancer by comparative genomic hybridization. Genes, Chrom. Cancer 12: 213-219.

Kamb, A., N .A. Gruis, J. Weaver-Fel dhaus, Q. Lui, K. Harshman, S.V. Tavigian, E. Stoker, R.S. Day, B.E. Johnson, and M.H. Skolnik. 1994. A cell cycle regulator potentially involved in genesis of many tumor types. Science 264: 463-440.

Kannio, A., M. Ridanpaa, H. Koskinen, T. Partanen, S. Anttila, Y. Collan, E. Hietanen, H. Vainio, and K. Husgafvel-Pursiainen. 1996. A molecular and epidemiological study on bladder cancer: p53 mutations, tobacco smoking and occupational exposure to asbestos. Cancer Epidemiol. Biomarkers Prev. 5: 33-39.

Knowles, M.A., P.A. Elder, M. Williamson, J.P. Cairns, M.E. Shaw, and M.G Law. 1994. Allel otype of human bladder cancer. Cancer Res. 54: 531-538.

Lowe, S.W. and T. Jacks. 1997. Response to a letter. Nature 385: 124-125.

Lukas, J., D. Perry, L. Aagaard, D.J. Mann, A. Bartokva, M. Strauss, G. Peters, and J. Bartek. 1995. Retinoblastoma-protein-dependent cell cycle inhibition by the tumor suppressor p16. Nature 375: 503-506.

M edema, R.H., R.E. Merrera, F. Lam, and R.A. Weinberg. 1995. Growth suppression by pl6ink4 requires functional retinoblastoma protein. Proc. Natl. Acad. Sci. 92: 6289-6293.

Merlo, A., J.G. Herman, L. Mao, D.J. Lee, E. Gabrielson, P.C. Burger, S.B. Baylin, and D. Sidransky. 1995. 5' CpG island methylation is associated with transcriptional silencing of the tumor suppressor p16/CDKN2/MTS1 in human cancers. Nature Med. 1: 686-692.

Mertens, F., B. Johansson, M. Hoglund, and F. Mitelman. 1997. Chromosome imbalance maps of malignant solid tumors: A cytologic survey of 3185 neoplasims. Cancer Res. 57: 27652780.

Meyerson, M., C.M. Counter, E.N. Eaton, L.W. Ellisen, P. Steiner, S.D. Caddle, L. Ziaugra, R.L. Beijersbergen, M.J. Davidoff, Q. Liu, S. Bacchetti, D.A. Haber, and R.A. Weinberg. 1997. hEST 2, the putative human tel omerase catalytic subunit gene, is up-regulated in tumor cells and during immortalization. Cell 90: 785-795.

Newton, M.A., S.Q. Wu, and C.A. Reznikoff. 1994. Assessing the significance of chromosome-loss data: Where are the suppressor genes for bladder cancer? Stat. Med. 13: 839-858.

N seyo, U.O. and D.L. Lamm. 1996. Therapy of superficial bladder cancer. Semin. Oncol. 23: 598-604.

Ohta, K., M. Kuwahara, N. Fujisaki, S. Kagawa, M. Fuihata, and Y. Ohtsuki. 1997. Overexpressed cyclin D1 protein related to $\mathrm{p} 53$ and retinoblastoma proteins in translational cell car- cinoma of the urinary tract. Oncology Rep. 4: 97-101.

Okajima, J., T. Fukuda, S. Okita, M.Tsutsumi, Y. Hirao, and Y. Konishi. 1996. Infrequent somatic alteration of p16/MTS1 in human primary superficial bladder cancers. Cancer Lett. 103: 227-231.

Presti, J.C., V.E. Reuter, T. Galan, W.R. Fair, and C. CordonCordo. 1991. M olecular genetic alterations in superficial and locally advanced human bladder cancer. Cancer Res. 51: 5405-5409.

Reznikoff, C.A., K.W. Gilchrist, D.H. Norback, K.B. Cummings, E. Erturk, and G.T. Bryan. 1983. Altered growth patterns in vitro of human papillary transitional carcinoma cells. Am. J. Pathol. 111: 263-272.

Reznikoff, C.A., L.J. Loretz, D.M. Presciotta, T.D. Oberley, and M.M. Ignatovic. 1987. Growth kinetics and differentiation in vitro of normal human uroepithelial cells on collagen gel substrates in defined medium. J. Cell Physiol. 131: 285-301.

Reznikoff, C.A., C. Belair, E. Savelieva, Y. Zhai, K. Pfeifer, T. Yeager, K.J. Thompson, S. DeVries, C. Bindley, M.A. N ewton, G. Sekhon, and F. Waldman. 1994. Long-term genome stability and minimal genotypic and phenotypic alterations in HPV16 E7-, but not E6-, immortalized human uroepithelial cells. Genes \& Dev. 8: 2227-2240.

Reznikoff, C.A., C.D. Belair, T.R. Yeager, E. Savelieva, R.H. Blelloch, J.A. Puthenveettil, and S. Cuthill. 1996a. A molecular genetic model of human bladder cancer pathogenesis. Semin. Oncol. 23: 571-584.

Reznikoff, C.A., T.R. Yeager, C.D. Belair, E. Savelieva, J.A. Puthenveettil, and W.M. Stadler. 1996b. Elevated p16 at senescence and loss of p16 at immortalization in human papillomavirus $16 \mathrm{E6}$, but not E7, transformed human urothelial cells. Cancer Res. 56: 2886-2890.

Ross, K., P.A. Jones, and M.C. Yu. 1996. Bladder cancer epidemiology and pathogenesis. Semin. Oncol. 23: 536-545.

Sarkis, A.S., G. Dalbagni, C. Cordon-Cordo, Z-F. Zhang, J. Sheinfeld, W.F. Fair, H.W. Herr, and V.E. Reuter. 1993. $\mathrm{N}$ uclear overexpression of p53 protein in transitional cell bladder carcinoma: A marker for disease progression. J. N atl. Cancer Inst. 85: 53-59.

Savelieva, E., C.D. Belair, M.A. N ewton, S. DeVries, J.W. Gray, F. Waldman, and C.A. Reznikoff. 1997. 20q gain associates with immortalization: 20q13.2 amplification correlates with genome instability in human papillomavirus 16 E7 transformed human uroepithelial cells. Oncogene 14: 551-560.

Serrano, M., G.J. Hannon, and D. Beach. 1993. A new regulatory motif in cell cycle control causing specific inhibition of cyclin D/CDK4. Nature 366: 704-707.

Serrano, M., A.W. Lin, M.E. McCurrach, D. Beach, and S.W. Lowe. 1997. Oncogenic ras provokes premature cell senescence associated with accumulation of p53 and p16ink4a. Cell 88: 593-602.

Shin, K.Y., G. Kong, W.S. Kim, T.Y. Lee, Y.N. Woo, and J.D. Lee. 1997. Overexpression of cyclin D1 correlates with early recurrence in superficial bladder cancers. Br. J. Cancer 75: 1788-1792.

Soder, A.I., S.F. Hoare, S. M uir, J.J. Going, E.K. Parkinson, and W.N. Keither. 1997. Amplification, increased dosage and in situ expression of the tel omerase gene in human cancer. Oncogene 14: 1013-1021.

Spruck, C.H., P.F. Ohneseit, M. Gonzalez-Zuleta, D. Esrig, N. Miyao, Y.C. Tsai, S.P. Lerner, C. Schmutte, A.S. Yang, R. Cote, L. Dubeau, P.W. Nichols, G.G. Hermann, K. Steven, T. Horn, D.G. Skinner, and P.A. Jones. 1994a. Two molecular pathways to transitional cell carcinoma of the bladder. Cancer Res. 54: 784-788.

Spruck, C.H., M. Gonzalez-Zulueta, A. Shibata, A. Simoneau, 
Yeager et al.

M-F. Lin, F. Gonzales, Y.C. Tsai, and P.A. Jones. 1994b. p16 gene in uncultured tumors. Nature 370: 183-184.

Takle, L.A. and M.A. Knowles. 1996. Deletion mapping implicates two tumor suppressor genes on chromosome $8 p$ in the devel opment of bladder cancer. Oncogene 12: 1083-1087.

Vieten, L., C.D. Belair, L. Savelieva, K. Julicher, F. Broker, W. Bardenheuer, and J. Schuttee. 1997. Minimal deletion of $3 p 13 \rightarrow$ p14.2 associated with immortalization of human uroepithelial cells. Genes, Chrom. Cancer (in press).

Xu, H.-J., P. Cairns, S.-X. Hu, M .A. Knowles, and W.F. Benedict. 1993. Loss of RB protein expression in primary bladder cancer correl ates with loss of heterozygosity at the RB locus and tumor progression. Int. J. Cancer 53: 781-784.

Yeager, T., W. Stadler, C. Belair, J. Puthenveettil, O. Olopade, and C. Reznikoff. 1995. Increased p16 levels correlate with $\mathrm{pRb}$ alterations in human urothelial cells. Cancer Res. 55: 493-497. 


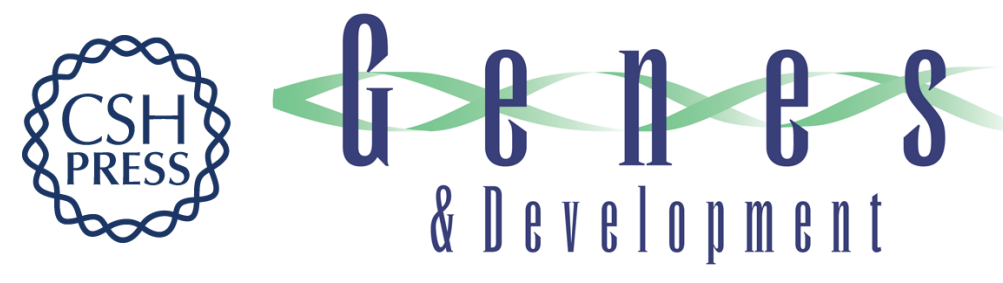

\section{Overcoming cellular senescence in human cancer pathogenesis}

Thomas R. Yeager, Sandy DeVries, David F. Jarrard, et al.

Genes Dev. 1998, 12:

Access the most recent version at doi:10.1101/gad.12.2.163

References This article cites 42 articles, 15 of which can be accessed free at: http://genesdev.cshlp.org/content/12/2/163.full.html\#ref-list-1

License

Email Alerting Receive free email alerts when new articles cite this article - sign up in the box at the top Service right corner of the article or click here.

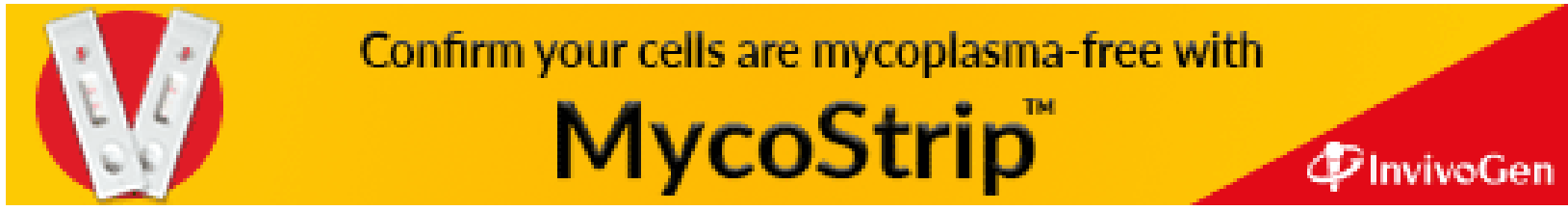

